\title{
Chebyshev tau meshless method based on the highest derivative for solving a class of two-dimensional parabolic problems
}

\author{
Wenting Shao ${ }^{1} \&$ Xionghua $\mathrm{Wu}^{1,2}$ \\ ${ }^{I}$ Department of Applied Mathematics, Tongji University, P. R. China \\ ${ }^{2} M P T C, X i$ 'an Jiaotong Liverpool University, P. R. China
}

\begin{abstract}
We propose a new method for the numerical solution of a class of twodimensional parabolic problems. Our algorithm is based on the use of the Alternating Direction Implicit (ADI) approach in conjunction with the Chebyshev tau meshless method based on the highest derivative (CTMMHD). CTMMHD is applied to solve the set of one-dimensional problems resulting from operator-splitting at each time-stage. CTMMHD-ADI yields spectral accuracy in space and second order in time. Several numerical experiments are implemented to verify the efficiency of our method.

Keywords: Chebyshev tau meshless method, the highest derivative, Alternating Direction Implicit, convection-diffusion problems, variable coefficients, nonlinear parabolic problems.
\end{abstract}

\section{Introduction}

In this paper, we present a new method, which combines the well-known Alternating Direction Implicit (ADI) method [1] with Chebyshev tau meshless method based on the highest derivative (CTMMHD) [2].

The tau approach is a kind of classical meshless method. In the previous study, we have proposed Chebyshev tau meshless method based on the highest derivative (CTMMHD) [2]. The starting point is the Chebyshev expansion of the highest derivative, and then the lower derivatives and the unknown function are constructed through an integration process. It is worthwhile to mention that for 
one dimensional problem, CTMMHD leads to the coefficients matrices with low magnitude condition numbers $\mathcal{O}(1)$.

Alternating Direction Implicit (ADI) approach is of interest since it can solve multi-dimensional problems as a series of one dimensional problems. Hence, there is a significant reduction in the computing time and storage requirements. Moreover, ADI algorithms can yield unconditional stability at approximately the same cost per time-step as explicit finite-difference formulations [3]. Various finite-difference-based ADI algorithms for unsteady convection-diffusion problems have been put forward [4-6]. ADI methods have also been previously used in conjunction with spatial differentiation methods which, do not rely on finite differencing in recent years, for example, Bruno and Lyon developed Fourier-Continuation Alternating-Direction (FC-AD) methodology [3].

In our method, the original two-dimensional problem is reduced to a system of Ordinary Differential Equations (ODEs) by the classical ADI approach [1]. To complete the time-stepping algorithm, each ODE is solved by CTMMHD. Our method yields spectral accuracy in space and second order in time. This paper is the first step of the application of CTMMHD-ADI for solving time dependent problems.

\section{Chebyshev approximation}

\subsection{Integration and multiplication of Chebyshev expansions}

The Chebyshev polynomial $T_{i}(x)$ satisfies the following property [7]

$$
\begin{gathered}
\int^{x} T_{0}(y) d y=T_{1}(x), \int^{x} T_{1}(y) d y=\frac{T_{2}(x)}{4}+\frac{T_{0}(x)}{4}, \\
\int^{x} T_{i}(y) d y=\frac{T_{i+1}(x)}{2(i+1)}-\frac{T_{i-1}(x)}{2(i-1)}, x \in[-1,1], i \geq 2 .
\end{gathered}
$$

Consider the truncated Chebyshev series of $u_{x x}(x), u_{x}(x), u(x)$ :

$$
u_{x x}(x)=\sum_{i=0}^{N-3} a_{i} T_{i}(x), u_{x}(x)=\sum_{i=0}^{N-3} b_{i} T_{i}(x), u(x)=\sum_{i=0}^{N-3} c_{i} T_{i}(x) .
$$

Denote $\mathbf{a}=\left[a_{i}\right]_{i=0}^{N-3}, \mathbf{b}=\left[b_{i}\right]_{i=0}^{N-3}, \mathbf{c}=\left[c_{i}\right]_{i=0}^{N-3}, \mathbf{U}=\left[c_{0}, b_{0}, a_{0}, \ldots, a_{N-3}\right]^{\mathrm{T}}$. Via property (1), we have

$$
\mathbf{a}=H_{0} \mathbf{U}, \mathbf{b}=H_{1} \mathbf{U}, \mathbf{c}=H_{2} \mathbf{U},
$$

here, $H_{i}, i=1,2,3$ are known integration matrices of dimension $(N-2) \times N$.

Considering the multiplication $V(x)=d(x) u(x)$, with Chebyshev coefficients $\mathbf{V}=\left[V_{i}\right]_{i=0}^{N-3}, \mathbf{d}=\left[d_{i}\right]_{i=0}^{N-3}$. We have the relationship between $\mathbf{V}$ and $\mathbf{U}$ in terms of $\mathbf{d}[8]$ :

$$
\mathbf{V}=M_{d} H_{2} \mathbf{U}, M_{d}=\left[M_{0} \mathbf{d}, M_{1} \mathbf{d}, \ldots, M_{N-3} \mathbf{d}\right],
$$

where 


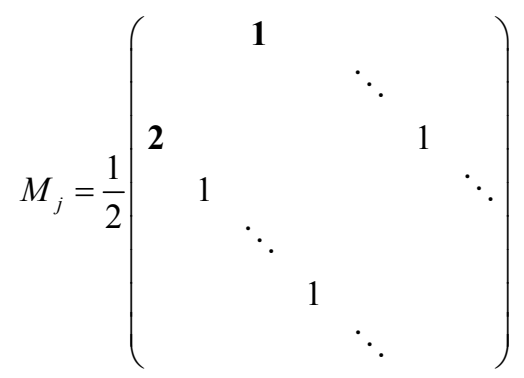

with the highlighted elements 2 at $(j+1,1)$ and $\mathbf{1}$ at $(1, j+1)$.

\section{CTMMHD-ADI algorithm and implementation}

\subsection{Splitting of the two-dimensional convection-diffusion equation}

We consider the following problem on $\Omega=[-1,1]^{2}$,

$$
\begin{gathered}
\frac{\partial u}{\partial t}=a \cdot u_{x x}+b \cdot u_{y y}-p \cdot u_{x}-q \cdot u_{y}+Q(x, y, t),(x, y, t) \in \Omega \times(0, T], \\
u(x, y, t)=G(x, y, t),(x, y) \in \partial \Omega, t \in(0, T], \\
u(x, y, 0)=u_{0}(x, y),(x, y) \in \Omega,
\end{gathered}
$$

where $G, u_{0}$ and the source term $Q$ are given sufficiently smooth functions. $p$ and $q$ are constant, convective velocities; $a$ and $b$ are constant, positive diffusion coefficients in the $x$ and $y$ direction, respectively.

Approximate the solution at time $t^{n}=n \Delta t, n \geq 0, \Delta t$ is the time increment, and let $u^{n}=u\left(x, y, t^{n}\right), Q^{n+1 / 2}=Q\left(x, y, t^{n+1 / 2}\right)$. Using a centre finite-difference scheme around $t^{n+1 / 2}=(n+1 / 2) \Delta t$, yields

$$
\begin{aligned}
& \frac{u^{n+1}-u^{n}}{\Delta t}=\frac{a}{2} \frac{\partial^{2}}{\partial x^{2}}\left(u^{n+1}+u^{n}\right)+\frac{b}{2} \frac{\partial^{2}}{\partial y^{2}}\left(u^{n+1}+u^{n}\right) \\
& \quad-\frac{p}{2} \frac{\partial}{\partial x}\left(u^{n+1}+u^{n}\right)-\frac{q}{2} \frac{\partial}{\partial y}\left(u^{n+1}+u^{n}\right)+Q^{n+1 / 2}(x, y, t)+E_{1}(x, y, \Delta t)
\end{aligned}
$$

where

$\left\|E_{1}(x, y, \Delta t)\right\| \leq \Delta t^{2} / 24\left\|u_{t t t}\right\|+\Delta t^{2} / 8\|a\| \cdot\left\|u_{t t x x}\right\|+\Delta t^{2} / 8\|b\| \cdot\left\|u_{t t y y}\right\|+\Delta t^{2} / 8\|p\| \cdot\left\|u_{t t x}\right\|$ $+\Delta t^{2} / 8\|q\| \cdot\left\|u_{t t y}\right\|,\|\cdot\|=\|\cdot\|_{L^{\infty}\left(\Omega \times\left(t^{n}, t^{n+1}\right)\right)} \cdot$

First, introduce the operators $R_{x}=1+\Delta t / 2\left(a \cdot \partial^{2} / \partial x^{2}-p \cdot \partial / \partial x\right), R_{y}=1+\Delta t / 2\left(b \cdot \partial^{2} / \partial y^{2}-q \cdot \partial / \partial y\right)$, $L_{x}=1-\Delta t / 2\left(a \cdot \partial^{2} / \partial x^{2}-p \cdot \partial / \partial x\right), L_{y}=1-\Delta t / 2\left(b \cdot \partial^{2} / \partial y^{2}-q \cdot \partial / \partial y\right)$, eqn. (5) becomes 


$$
L_{x} L_{y} u^{n+1}=R_{x} R_{y} u^{n}+\Delta t Q^{n+1 / 2}+E_{2}(x, y, \Delta t)+\Delta t E_{1}(x, y, \Delta t),
$$

where $\left\|E_{2}(x, y, \Delta t)\right\| \leq C \Delta t^{3}\left(\left\|u_{t x x y y}\right\|+\left\|u_{t x x y}\right\|+\left\|u_{t x y y}\right\|+\left\|u_{t x y}\right\|\right), C$ is a constant independent of $\Delta t$.

We introduce the approximation $Q^{n+1 / 2}=1 / 2\left(R_{x} Q^{n+1 / 4}+L_{x} Q^{n+3 / 4}\right)+E_{3}(x$, $y, \Delta t), \quad$ with $\left\|E_{3}(x, y, \Delta t)\right\| \leq \Delta t^{2} / 16\left\|Q_{t t}\right\|+\Delta t^{2} / 8\|a\| \cdot\left\|Q_{t x x}\right\|+\Delta t^{2} / 8\|p\| \cdot\left\|Q_{t x}\right\|$, and establish a scheme of the form

$$
\begin{gathered}
L_{x} \tilde{u}^{n+1 / 2}=R_{y} \tilde{u}^{n}+\Delta t / 2 \cdot Q^{n+1 / 4}, \\
L_{y} \tilde{u}^{n+1}=R_{x} \tilde{u}^{n+1 / 2}+\Delta t / 2 \cdot Q^{n+3 / 4},
\end{gathered}
$$

where $\tilde{u}^{n}$ is the approximation to the exact solution $u^{n}$.

Eqn. (7) predicts a second order accurate in time [3].

In order to facilitate the description of our algorithm, we introduce the new variables

$$
w^{n+1 / 2}=R_{x} \tilde{u}^{n+1 / 2}, w^{n+1}=R_{y} \tilde{u}^{n+1} .
$$

Let $f^{n+1 / 4}=w^{n}+\Delta t / 2 \cdot Q^{n+1 / 4}$ and $f^{n+3 / 4}=w^{n+1 / 2}+\Delta t / 2 \cdot Q^{n+3 / 4}$, then eqn. (7) is equivalent to

$$
\begin{gathered}
L_{x} \tilde{u}^{n+1 / 2}(x, y)=f^{n+1 / 4}(x, y), \\
L_{y} \tilde{u}^{n+1}(x, y)=f^{n+3 / 4}(x, y) .
\end{gathered}
$$

After some manipulations, we obtain

$$
\begin{gathered}
w^{n+1 / 2}=2 \tilde{u}^{n+1 / 2}-f^{n+1 / 4}, \\
w^{n+1}=2 \tilde{u}^{n+1}-f^{n+3 / 4} .
\end{gathered}
$$

Above equations have equivalent matrix form

$$
\begin{aligned}
& \left(H_{2}-\frac{a \Delta t}{2} H_{0}+\frac{p \Delta t}{2} H_{1}\right) \mathbf{U}^{n+1 / 2}=H_{2} \mathbf{F}^{n+1 / 4}, \\
& \mathbf{U}^{n+1}\left(H_{2}^{\mathrm{T}}-\frac{b \Delta t}{2} H_{0}^{\mathrm{T}}+\frac{q \Delta t}{2} H_{1}^{\mathrm{T}}\right)=\mathbf{F}^{n+3 / 4} H_{2}^{\mathrm{T}},
\end{aligned}
$$

and similarly

$$
\begin{aligned}
\mathbf{W}^{n+1 / 2} & =2 \mathbf{U}^{n+1 / 2}-\mathbf{F}^{n+1 / 4}, \\
\mathbf{W}^{n+1} & =2 \mathbf{U}^{n+1}-\mathbf{F}^{n+3 / 4},
\end{aligned}
$$

with Chebyshev coefficients matrices $\mathbf{U}^{n+1 / 2}, \mathbf{U}^{n+1}$ corresponding to $\tilde{u}^{n+1 / 2}, \tilde{u}^{n+1}$, respectively. Similar for $\mathbf{W}^{n+1 / 2}, \mathbf{W}^{n+1}, \mathbf{F}^{n+1 / 4}$ and $\mathbf{F}^{n+3 / 4}$.

\subsection{Treatment of boundary conditions}

For solving eqn. (10a), we consider the $\tilde{u}(x, y, t)$ at time $t=t^{n+1 / 2}$, which satisfies the boundary conditions

$$
\tilde{u}^{n+1 / 2}(1, y)=G\left(1, y, t^{n+1 / 2}\right), \tilde{u}^{n+1 / 2}(-1, y)=G\left(-1, y, t^{n+1 / 2}\right) .
$$


With the block matrix technique, denote $\mathbf{U}^{n+1 / 2}=\left[\begin{array}{c}\mathbf{U}_{1}^{n+1 / 2} \\ \mathbf{U}_{2}^{n+1 / 2}\end{array}\right]$, where

$$
\begin{aligned}
\mathbf{U}_{1}^{n+1 / 2} & =\left[\begin{array}{lllll}
c c_{0,0} & c b_{0,0} & c a_{0,0} & \cdots & c a_{0, N-3} \\
b c_{0,0} & b b_{0,0} & b a_{0,0} & \cdots & b a_{0, N-3}
\end{array}\right], \\
\mathbf{U}_{2}^{n+1 / 2} & =\left[\begin{array}{ccccc}
a c_{0,0} & a b_{0,0} & a a_{0,0} & \cdots & a a_{0, N-3} \\
\vdots & \vdots & \vdots & \ddots & \vdots \\
a c_{N-3,0} & a b_{N-3,0} & a a_{N-3,0} & \cdots & a a_{N-3, N-3}
\end{array}\right] .
\end{aligned}
$$

Let

$$
\begin{aligned}
& P_{1}(y)=\frac{1}{2}\left(\tilde{u}_{y y}^{n+1 / 2}(1, y)-\tilde{u}_{y y}^{n+1 / 2}(-1, y)\right), P_{2}(y)=\frac{1}{2}\left(\tilde{u}_{y y}^{n+1 / 2}(1, y)+\tilde{u}_{y y}^{n+1 / 2}(-1, y)\right), \\
& P_{3}(y)=\frac{1}{2}\left(\tilde{u}_{y}^{n+1 / 2}(1, y)-\tilde{u}_{y}^{n+1 / 2}(-1, y)\right), P_{4}(y)=\frac{1}{2}\left(\tilde{u}_{y}^{n+1 / 2}(1, y)+\tilde{u}_{y}^{n+1 / 2}(-1, y)\right), \\
& P_{5}(y)=\frac{1}{2}\left(\tilde{u}^{n+1 / 2}(1, y)-\tilde{u}^{n+1 / 2}(-1, y)\right), P_{4}(y)=\frac{1}{2}\left(\tilde{u}^{n+1 / 2}(1, y)+\tilde{u}^{n+1 / 2}(-1, y)\right),
\end{aligned}
$$

with the Chebyshev coefficients $\mathbf{P}_{j}=\left[P_{i, j}\right]_{i=0}^{N-3}, j=1,2, \ldots, 6$.

Based on the boundary condition treatment described in [2, Sec.3.1], we arrive at

$$
\mathbf{U}_{1}^{n+1 / 2}=W_{1} \mathbf{U}_{2}^{n+1 / 2}+g_{1},
$$

with

$$
W_{1}=\left[\begin{array}{c}
Q_{2} \\
Q_{1}
\end{array}\right], g_{1}=\left[\begin{array}{ccc}
P_{1,6} & P_{1,4} & \mathbf{P}_{2}^{\mathrm{T}} \\
P_{1,5} & P_{1,3} & \mathbf{P}_{1}^{\mathrm{T}}
\end{array}\right] \text {. }
$$

Now, denote $L H S^{n+1 / 2}=H_{2}-a \Delta t / 2 \cdot H_{0}+p \Delta t / 2 \cdot H_{1}, \quad L H S_{1}^{n+1 / 2}=L H S^{n+1 / 2}$ $(:, 1: 2)$ and $L H S_{2}^{n+1 / 2}=L H S^{n+1 / 2}(:, 3:$ end $)$, thus eqn. (10a) is equivalent to:

$$
\left(L H S_{1}^{n+1 / 2} W_{1}+L H S_{2}^{n+1 / 2}\right) \mathbf{U}_{2}^{n+1 / 2}=H_{2} \mathbf{F}^{n+1 / 4}-L H S_{1}^{n+1 / 2} g_{1} \text {. }
$$

Similar for solving eqn. (10b), which is defined at time $t=t^{n+1}$, and satisfies the boundary conditions

$$
\tilde{u}^{n+1}(x, 1)=G\left(x, 1, t^{n+1}\right), \tilde{u}^{n+1}(x,-1)=G\left(x,-1, t^{n+1}\right) .
$$

We denote $\mathbf{U}^{n+1}=\left[\mathbf{U}_{3}^{n+1}, \mathbf{U}_{4}^{n+1}\right]$, with

$$
\mathbf{U}_{3}^{n+1}=\left[\begin{array}{cc}
c c_{0,0} & c b_{0,0} \\
b c_{0,0} & b b_{0,0} \\
a c_{0,0} & a b_{0,0} \\
\vdots & \vdots \\
\vdots & \vdots \\
a c_{N-3,0} & a b_{N-3,0}
\end{array}\right], \mathbf{U}_{4}^{n+1}=\left[\begin{array}{cccc}
c a_{0,0} & \cdots & \cdots & c a_{0, N-3} \\
b a_{0,0} & \cdots & \cdots & b a_{0, N-3} \\
a a_{0,0} & \cdots & \cdots & a a_{0, N-3} \\
\vdots & \ddots & & \vdots \\
\vdots & & \ddots & \vdots \\
a a_{N-3,0} & \cdots & \cdots & a a_{N-3, N-3}
\end{array}\right] .
$$


Let

$$
\begin{aligned}
& P_{7}(x)=\frac{1}{2}\left(\tilde{u}_{x x}^{n+1}(x, 1)-\tilde{u}_{x x}^{n+1}(x,-1)\right), P_{8}(x)=\frac{1}{2}\left(\tilde{u}_{x x}^{n+1}(x, 1)+\tilde{u}_{x x}^{n+1}(x,-1)\right), \\
& P_{9}(x)=\frac{1}{2}\left(\tilde{u}_{x}^{n+1}(x, 1)-\tilde{u}_{x}^{n+1}(x,-1)\right), P_{10}(x)=\frac{1}{2}\left(\tilde{u}_{x}^{n+1}(x, 1)+\tilde{u}_{x}^{n+1}(x,-1)\right), \\
& P_{11}(x)=\frac{1}{2}\left(\tilde{u}^{n+1}(x, 1)-\tilde{u}^{n+1}(x,-1)\right), P_{12}(x)=\frac{1}{2}\left(\tilde{u}^{n+1}(x, 1)+\tilde{u}^{n+1}(x,-1)\right),
\end{aligned}
$$

with the Chebyshev coefficients $\mathbf{P}_{j}=\left[P_{i, j}\right]_{i=0}^{N-3}, j=7,8, \ldots, 12$.

We similarly have

$$
\mathbf{U}_{3}^{n+1}=\mathbf{U}_{4}^{n+1} W_{2}+g_{2},
$$

with

$$
W_{2}=\left[Q_{2}^{\mathrm{T}}, Q_{1}^{\mathrm{T}}\right], g_{2}=\left[\begin{array}{cc}
P_{1,12} & P_{1,11} \\
P_{1,10} & P_{1,9} \\
\mathbf{P}_{8} & \mathbf{P}_{7}
\end{array}\right] .
$$

Denote $L H S^{n+1}=H_{2}^{\mathrm{T}}-b \Delta t / 2 \cdot H_{0}^{\mathrm{T}}+q \Delta t / 2 \cdot H_{1}^{\mathrm{T}}, \quad \quad L H S_{3}^{n+1}=L H S^{n+1}(1: 2,:)$ and $L H S_{4}^{n+1}=L H S^{n+1}(3:$ end,$:)$. Eqn. (10b) is equivalent to

$$
\mathbf{U}_{4}^{n+1}\left(W_{2} L H S_{3}^{n+1}+L H S_{4}^{n+1}\right)=\mathbf{F}^{n+3 / 4} H_{2}^{\mathrm{T}}-g_{2} L H S_{3}^{n+1} .
$$

\subsection{Numerical methods for the problem with variable coefficients}

Considering the initial-boundary value problem with variable coefficients

$$
\frac{\partial u}{\partial t}=L u+Q(x, y, t),(x, y, t) \in \Omega \times(0, T]
$$

where the boundary and initial conditions are given in eqn. (4b) and (4c). The elliptic operator is given by

$$
L u=a(x, y) \frac{\partial^{2} u}{\partial x^{2}}+b(x, y) \frac{\partial^{2} u}{\partial y^{2}}-p(x, y) \frac{\partial u}{\partial x}-q(x, y) \frac{\partial u}{\partial y}-c(x, y) u,
$$

with known smooth functions $a(x, y), b(x, y), p(x, y), q(x, y)$ and $c(x, y)$.

Inspired by the idea in eqn. (7), we take an iteration algorithm

$$
\begin{gathered}
\left(1-\frac{a\left(x, y_{0}\right) \Delta t}{2} \frac{\partial^{2}}{\partial x^{2}}+\frac{p\left(x, y_{0}\right) \Delta t}{2} \frac{\partial}{\partial x}+\frac{c\left(x, y_{0}\right) \Delta t}{4}\right) \tilde{u}^{n+1 / 2, k+1}=f^{n+1 / 4} \\
+\left(\frac{a(x, y) \Delta t}{2}-\frac{a\left(x, y_{0}\right) \Delta t}{2}\right) \frac{\partial^{2}}{\partial x^{2}} \tilde{u}^{n+1 / 2, k}-\left(\frac{p(x, y) \Delta t}{2}-\frac{p\left(x, y_{0}\right) \Delta t}{2}\right) \frac{\partial}{\partial x} \tilde{u}^{n+1 / 2, k} \\
-\left(\frac{c(x, y) \Delta t}{4}-\frac{c\left(x, y_{0}\right) \Delta t}{4}\right) \tilde{u}^{n+1 / 2, k}
\end{gathered}
$$

where $f^{n+1 / 4}=\left(1+b(x, y) \Delta t / 2 \cdot \partial^{2} / \partial y^{2}-q(x, y) \Delta t / 2 \cdot \partial / \partial y-c(x, y) \Delta t / 4\right) \tilde{u}^{n}+\Delta t / 2$ $\cdot Q^{n+1 / 4}, \tilde{u}^{n+1 / 2,0}=\tilde{u}^{n}$, and $\forall y_{0} \in[-1,1]$, to simplify, take $y_{0}=-1$. 
If $\left|\tilde{u}^{n+1 / 2, k+1}-\tilde{u}^{n+1 / 2, k}\right| \geq \operatorname{tol}($ tol $:$ the tolerance error), let $k=k+1, k=0,1$, $2, \ldots$; otherwise, denote $\tilde{u}^{n+1 / 2}=\tilde{u}^{n+1 / 2, k+1}$ and go to another direction

$$
\begin{gathered}
\left(1-\frac{b\left(x_{0}, y\right) \Delta t}{2} \frac{\partial^{2}}{\partial y^{2}}+\frac{q\left(x_{0}, y\right) \Delta t}{2} \frac{\partial}{\partial y}+\frac{c\left(x_{0}, y\right) \Delta t}{4}\right) \tilde{u}^{n+1, k+1}=f^{n+3 / 4} \\
+\left(\frac{b(x, y) \Delta t}{2}-\frac{b\left(x_{0}, y\right) \Delta t}{2}\right) \frac{\partial^{2}}{\partial y^{2}} \tilde{u}^{n+1, k}-\left(\frac{q(x, y) \Delta t}{2}-\frac{q\left(x_{0}, y\right) \Delta t}{2}\right) \frac{\partial}{\partial y} \tilde{u}^{n+1, k} \\
-\left(\frac{c(x, y) \Delta t}{4}-\frac{c\left(x_{0}, y\right) \Delta t}{4}\right) \tilde{u}^{n+1, k}
\end{gathered}
$$

where

$f^{n+3 / 4}=\left(1+a(x, y) \Delta t / 2 \cdot \partial^{2} / \partial x^{2}-p(x, y) \Delta t / 2 \cdot \partial / \partial x-c(x, y) \Delta t / 4\right) \tilde{u}^{n+1 / 2}+$ $\Delta t / 2 \cdot Q^{n+3 / 4}, \tilde{u}^{n+1,0}=\tilde{u}^{n+1 / 2}$ and $x_{0}=-1$.

If $\left|\tilde{u}^{n+1, k+1}-\tilde{u}^{n+1, k}\right| \geq$ tol, $\quad$ let $k=k+1, k=0,1,2, \ldots ; \quad$ otherwise, denote $\tilde{u}^{n+1}=\tilde{u}^{n+1, k+1}$ and go to eqn. (20) at next time step.

\section{Numerical experiments}

We demonstrate the applicability of CTMMHD-ADI through some numerical tests. Given the numerical solution $u^{\text {num }}$ and the exact solution $u^{\text {exa }}$, the following errors are considered

$$
L^{\infty}=\max _{1 \leq i \leq \tilde{N}}\left|u_{i}^{\text {exa }}-u_{i}^{\text {num }}\right|, L^{2}=\sqrt{\sum_{i=1}^{\tilde{N}}\left|u_{i}^{\text {exa }}-u_{i}^{\text {num }}\right|^{2}},
$$

where $\tilde{N}$ is the number of test nodes calculated. The boundary and initial conditions are directly taken from the analytical solutions, and the initial guess is zero.

Problem 5.1 Consider the steady-state convection-diffusion problem [9],

$$
\begin{gathered}
-\left(\frac{\partial^{2} u}{\partial x^{2}}+\frac{\partial^{2} u}{\partial y^{2}}\right)+\operatorname{Re} \frac{\partial u}{\partial x}=0,0 \leq x, y \leq 1, \\
u(x, 0)=0, u(x, 1)=0,0 \leq x \leq 1, \\
u(0, y)=\sin (\pi y), u(1, y)=2 \sin (\pi y), 0 \leq y \leq 1,
\end{gathered}
$$

with the exact solution

$$
u(x, y)=\exp (\operatorname{Re} x / 2) \sin (\pi y) \frac{2 \exp (-\mathrm{Re} / 2) \sinh \sigma x+\sinh \sigma(1-x)}{\sinh \sigma},
$$

where $\sigma=\sqrt{\pi^{2}+\operatorname{Re}^{2} / 4}$. This problem produces a layer along the line $x=1$, but convection is limited to the $x$ direction only (Figure. 1 (a) : $\operatorname{Re}=10^{3}$ ). 
In the case with our meshless method, firstly transform the original domain into $(\tilde{x}, \tilde{y}) \in[-1,1]^{2}$. Secondly, introduce the $\sinh$ transform proposed by Tee and Trefethen [10] to reduce the singularity in $\tilde{x}$ direction,

$$
\tilde{x}=\delta+\kappa \sinh \left[\left(\sinh ^{-1}\left(\frac{1-\delta}{\kappa}\right)+\sinh ^{-1}\left(\frac{1+\delta}{\kappa}\right)\right) \frac{\xi-1}{2}+\sinh ^{-1}\left(\frac{1-\delta}{\kappa}\right)\right],
$$

where $\delta, \kappa$ are parameters dependent on the singularity of the solution, which respectively represent the location and width of the boundary layer. $\kappa=c \varepsilon, \varepsilon$ is a small parameter and $c$ is an appropriate chosen constant. For this problem, $\varepsilon=2 / \operatorname{Re}, c=2$.

The transformed equation

$$
\bar{a} v_{\xi \xi}+\bar{b} v_{\tilde{y} \tilde{y}}+\bar{c} v_{\xi}=0
$$

where $v$ is the transplant of $u$ and the transformed coefficients

$$
\bar{a}=-\varepsilon \xi_{\tilde{x}}^{2}, \bar{b}=-\varepsilon, \bar{c}=-\varepsilon \xi_{\tilde{x} \tilde{x}}+\xi_{\tilde{x}} .
$$

We take $\operatorname{Re}=1,10,10^{2}, 10^{3}$ for testing, and $\Delta t=0.5$. Figure. 1 (b) shows the $L^{2}$ norm errors with different Chebyshev series numbers, which illustrates the exponential convergence rate in space of our method.

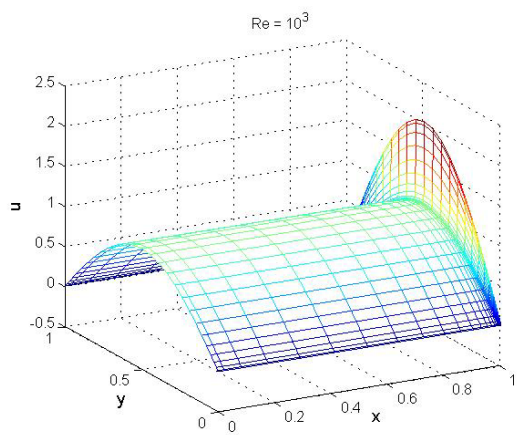

(a): The numerical solution, $N-2=29$

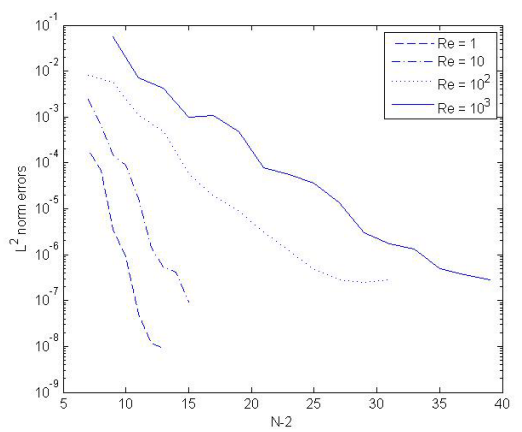

(b): The convergence rate in space

\section{Figure 1: $\quad$ Problem 5.1.}

Problem 5.2 Consider the two-dimensional non-linear viscous Burgers' equation [11] on $t \in[0,1.25], \varepsilon=0.05$

$$
\frac{\partial u}{\partial t}+u \frac{\partial u}{\partial x}+u \frac{\partial u}{\partial y}=\varepsilon\left(\frac{\partial^{2} u}{\partial x^{2}}+\frac{\partial^{2} u}{\partial y^{2}}\right), 0 \leq x, y \leq 1 .
$$

Subject to initial and boundary conditions, the exact transient solution is derived as

$$
u(x, y, t)=\left[1+\exp \left(\frac{x+y-t}{2 \varepsilon}\right)\right]^{-1}
$$


After the computational domain transformed into $(\tilde{x}, \tilde{y}) \in[-1,1]^{2}$, the problem is solved by the linearization process as follows

$$
\begin{array}{r}
\left(1-2 \Delta t \varepsilon \frac{\partial^{2}}{\partial \tilde{x}^{2}}+\Delta t \tilde{u}^{n+1 / 2, k}\left(\tilde{x}, \tilde{y}_{0}\right) \frac{\partial}{\partial \tilde{x}}\right) \tilde{u}^{n+1 / 2, k+1}=\left(1+2 \Delta t \varepsilon \frac{\partial^{2}}{\partial \tilde{y}^{2}}-\Delta t \tilde{u}^{n}(\tilde{x}, \tilde{y}) \frac{\partial}{\partial \tilde{y}}\right) \tilde{u}^{n} \\
+\Delta t\left(\tilde{u}^{n+1 / 2, k}\left(\tilde{x}, \tilde{y}_{0}\right)-\tilde{u}^{n+1 / 2, k}(\tilde{x}, \tilde{y})\right) \frac{\partial}{\partial \tilde{x}} \tilde{u}^{n+1 / 2, k}, \\
\left(1-2 \Delta t \varepsilon \frac{\partial^{2}}{\partial \tilde{y}^{2}}+\Delta t \tilde{u}^{n+1, k}\left(\tilde{x}_{0}, \tilde{y}\right) \frac{\partial}{\partial \tilde{y}}\right) \tilde{u}^{n+1, k+1} \\
=\left(1+2 \Delta t \varepsilon \frac{\partial^{2}}{\partial \tilde{x}^{2}}-\Delta t \tilde{u}^{n+1 / 2}(\tilde{x}, \tilde{y}) \frac{\partial}{\partial \tilde{x}}\right) \tilde{u}^{n+1 / 2} \\
+\Delta t\left(\tilde{u}^{n+1, k}\left(\tilde{x}_{0}, \tilde{y}\right)-\tilde{u}^{n+1, k}(\tilde{x}, \tilde{y})\right) \frac{\partial}{\partial \tilde{y}} \tilde{u}^{n+1, k},
\end{array}
$$

with $\tilde{x}_{0}=\tilde{y}_{0}=-1$.

Figure. 2(a) depicts the $L^{\infty}$ norm errors at each time level with various time steps, $N-2=20, t o l=1 e-10$, and the numerical solution at $T=1.25$ is plotted in Figure. 2(b) . Karaa [11] solve this example by the High Order Compact ADI method. It is concluded that with $\Delta x=\Delta y=0.05$ and $\Delta t=1.00 \times 10^{-3}$, the $L^{\infty}$ norm error is $0.0042 ; \Delta t=5.00 \times 10^{-4}$, the $L^{\infty}$ norm error is improved to 0.0024 . Finally, it is improved into $1.5382 e-004$ with $\Delta x=\Delta y=0.025$, $\Delta t=1.00 \times 10^{-5}$. However, our method obtains much better results with fewer unknowns.

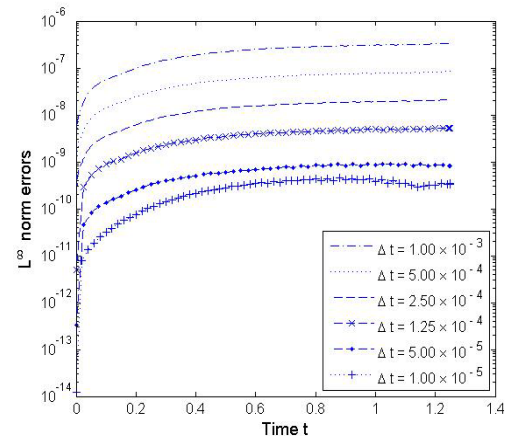

(a): The $L^{\infty}$ norm errors at each time level with various time step

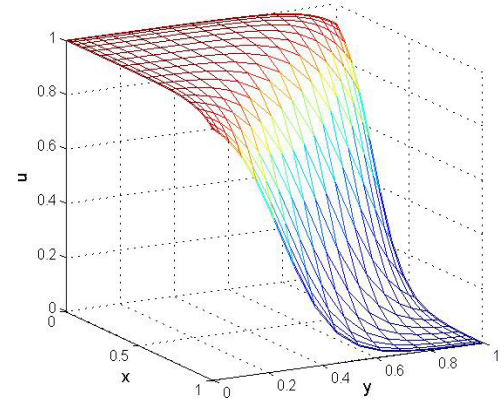

(b): The numerical solution at $T=1.25, \Delta t=1.00 \times 10^{-5}$

Figure 2: $\quad$ Problem 5.2.

Problem 5.3 Consider the system of two-dimensional Burgers' equations [12],

$$
\begin{gathered}
u_{t}+u u_{x}+v u_{y}=\varepsilon\left(u_{x x}+u_{y y}\right), 0 \leq x, y \leq 1, \\
v_{t}+u v_{x}+v v_{y}=\varepsilon\left(v_{x x}+v_{y y}\right), 0 \leq x, y \leq 1,
\end{gathered}
$$

with the exact solutions 


$$
\begin{aligned}
& u(x, y, t)=\frac{3}{4}-\frac{1}{4[1+\exp ((-4 x+4 y-t) /(32 \varepsilon))]}, \\
& v(x, y, t)=\frac{3}{4}+\frac{1}{4[1+\exp ((-4 x+4 y-t) /(32 \varepsilon))]} .
\end{aligned}
$$

Khater et al. [12] combined traditional Chebyshev Spectral Collocation method (ChSC) with Runge-Kutta method of order four to solve this problem. Compared with the results obtained by ChSC (table 1), CTMMHD-ADI gives higher accuracy (table 2).

Table 1: $\quad$ The $L^{\infty}$ norm error of $u$ and $v$ for ChSC.

\begin{tabular}{|c|c|c|c|c|c|c|}
\hline \multicolumn{3}{|c|}{ ChSC $[12]$} & \multicolumn{2}{c|}{$T=0.01$} & \multicolumn{2}{c|}{$T=2$} \\
\hline$\varepsilon$ & $\Delta t$ & $N_{p}+1$ & $u$ & $v$ & $u$ & $v$ \\
\hline 1.000 & $5.00 \times 10^{-4}$ & 11 & $1.31 e-06$ & $1.91 e-06$ & $1.61 e-06$ & $1.91 e-06$ \\
\hline 0.100 & $5.00 \times 10^{-3}$ & 11 & $4.77 e-07$ & $5.96 e-06$ & $1.13 e-06$ & $1.97 e-06$ \\
\hline 0.010 & $1.00 \times 10^{-3}$ & 21 & $3.22 e-06$ & $4.53 e-06$ & $1.49 e-05$ & $1.03 e-05$ \\
\hline 0.005 & $1.00 \times 10^{-3}$ & 31 & $2.25 e-05$ & $2.19 e-05$ & $9.99 e-05$ & $1.06 e-04$ \\
\hline
\end{tabular}

$N_{p}+1$ : the number of Chebyshev collocation points

Table 2: $\quad$ The $L^{\infty}$ norm error of $u$ and $v$ for CTMMHD-ADI.

\begin{tabular}{|c|c|c|c|c|c|c|}
\hline \multicolumn{3}{|c|}{ CTMMHD-ADI } & \multicolumn{2}{c|}{$T=0.01$} & \multicolumn{2}{c|}{$T=2$} \\
\hline$\varepsilon$ & $\Delta t$ & $N-2$ & $u$ & $v$ & $u$ & $v$ \\
\hline 1.000 & $5.00 \times 10^{-4}$ & 10 & $6.34 e-13$ & $6.31 e-13$ & $1.00 e-12$ & $1.00 e-12$ \\
\hline 0.100 & $5.00 \times 10^{-3}$ & 10 & $4.89 e-08$ & $4.89 e-08$ & $4.42 e-08$ & $4.42 e-08$ \\
\hline 0.010 & $1.00 \times 10^{-3}$ & 26 & $4.69 e-07$ & $4.69 e-07$ & $6.26 e-07$ & $6.26 e-07$ \\
\hline 0.005 & $1.00 \times 10^{-3}$ & 40 & $1.95 e-06$ & $1.95 e-06$ & $1.12 e-05$ & $1.12 e-05$ \\
\hline
\end{tabular}

\section{References}

[1] Peaceman, D.W. and H.H. Rachford, Jr., The numerical solution of parabolic and elliptic differential equations. Journal of the Society for Industrial and Applied Mathematics, 3(1), pp. 28-41, 1995.

[2] Shao, W. and Wu, X., Chebyshev tau meshless method based on the highest derivative for fourth order equations. Applied Mathematical Modelling, 37(3), pp. 1413-1430, 2013.

[3] Bruno, O.P. and Lyon, M., High-order unconditionally stable FC-AD solvers for general smooth domains I. Basic elements. Journal of Computational Physics, 229(6), pp. 2009-2033, 2010. 
[4] Karaa, S. and Zhang, J., High order ADI method for solving unsteady convection-diffusion problems. Journal of Computational Physics, 198(1), pp. 1-9, 2004.

[5] Spotz, W.F., High-order compact finite difference schemes for computational mechanics, Ph.D. Thesis. University of Texas at Austin: Austin, TX, 1995.

[6] Tian, Z.F. and Ge, Y.B., A fourth-order compact ADI method for solving two-dimensional unsteady convection-diffusion problems. Journal of Computational and Applied Mathematics, 198(1), pp. 268-286, 2007.

[7] Boyd, J.P., Chebyshev and Fourier Spectral Methods. Dover: New York, pp. 312-314, 2005.

[8] Kong, W. and Wu, X., Chebyshev tau matrix method for Poisson-type equations in irregular domain. Journal of Computational and Applied Mathematics, 228(1), pp. 158-167, 2009.

[9] E. C. Gartland, Jr., Discrete weighted mean approximation of a model convection-diffusion equation. SIAM Journal on Scientific and Statistical Computing, 3(4), pp. 460-472, 1982.

[10] Tee, T.W. and Trefethen, L.N., A rational spectral collocation method with adaptively transformed Chebyshev grid points. SIAM Journal on Scientific Computing, 28(5), pp. 1798-1811, 2006.

[11] Karaa, S., A high-order ADI method for parabolic problems with variable coefficients. International Journal of Computer Mathematics, 86(1), pp. 109-120, 2009.

[12] Khater, A.H., Temsah, R.S. and Hassan, M.M., A Chebyshev spectral collocation method for solving Burgers'-type equations. Journal of Computational and Applied Mathematics, 222(2), pp. 333-350, 2008. 